\title{
MÉTODO PARA LA FORMACIÓN DE STAKEHOLDER EN PROYECTOS DE INGENIERÍA USANDO LA METODOLOGÍA PMI Y TÉCNICAS DE INTELIGENCIA ARTIFICIAL
}

\author{
Luis Eduardo Vahos Hernández* \\ Danilo Mauricio Pastor Ramírez \\ Jovani Alberto Jiménez Builes ${ }^{* * *}$
}

Recibido: 22/05/2013

Aprobado: 25/10/2013

\section{RESUMEN}

En este artículo de investigación se presenta un método para la formación de interesados (stakeholder) en la ejecución de proyectos de ingeniería. El método integra investigaciones recientes en las áreas de la educación en ingeniería y técnicas de inteligencia artificial. El método es modelado a través de un sistema tutorial inteligente, el cual permite hacer un seguimiento cognitivo del interesado y del desarrollo del proyecto. También se utiliza el razonamiento basado en casos para ofrecer alternativas de solución cuando el stakeholder encuentra dificultades en la adquisición de los conocimientos.

Palabras clave: proyectos en ingeniería, interesados, elearning, planeación estratégica, entrenamiento de personal, inteligencia artificial.

M. Sc. Departamento de Ciencias de la Computación y de la Decisión, Facultad de Minas, Universidad Nacional de Colombia. Teléfono 57444884884.Correo levahosh@unal.edu.co. Autor para correspondencia.

** M. Sc. Escuela de Ingeniería de Sistemas, Facultad de Informática y Electrónica, Escuela Superior Politécnica del Chimborazo, Ecuador. Tel 593032998200. Correo: danilo.pastor@espoch.edu.ec

*** Ph. D. Departamento de Ciencias de la Computación y de la Decisión, Facultad de Minas, Universidad Nacional de Colombia. Teléfono 5744255222.Correo jajimen1@unal.edu.co 


\title{
METHOD FOR STAKEHOLDER FORMATION IN ENGINEERING PROJECTS USING THE PMI METHODOLOGY AND ARTIFICIAL INTELLIGENCE TECHNIQUES
}

\begin{abstract}
This research article shows a method for those persons (stakeholders) interested in executing engineering projects. The method integrates recent research works in the fields of education in engineering and artificial intelligence techniques. The method is modeled through an intelligent tutorial system which allows performing a cognitive monitoring of the interested person and the project development as well. Also, reasoning based on cases is used as a way to offer solution alternatives when stakeholders find difficulties to acquire knowledge.
\end{abstract}

Key words: Engineering projects; stakeholders; e-learning; strategic planning, staff training; artificial intelligence. 


\section{INTRODUCCIÓN}

Las estadísticas a nivel mundial avaladas por firmas encargadas de la medición de los proyectos en ingeniería, indican que solo el $22 \%$ de los mismos cumplen con todas las expectativas de los interesados (stakeholder). Esto se lo podemos atribuir a la poca educación que tiene los stakeholder en ejecución de proyectos. El éxito de los proyectos no solo se basa en el nivel de gestión de los administradores del mismo, si no que requiere que todos los stakeholder lo apoyen y participen activamente [1].

Los stakeholder son un factor importante en el éxito de los proyectos en ingeniería. La revisión de la literatura y la experiencia indican que los stakeholder no son capaces de interactuar de una manera ordenada y no tienen una relación armónica. Este es un factor importante en la insuficiencia de los proyectos. La cooperación de las partes interesadas tiene un impacto positivo importante en el éxito del mismo. La causa fundamental de fracaso de los proyectos son los intereses de sus participantes [2], [3].

La educación es la base del desarrollo del ser humano. Cuando se cuenta con el conocimiento y las competencias, el desarrollar nuestras actividades se realiza con un mejor desempeño logrando, de esta manera, una buena educación de nuestros interesados, y logrando un mejor entendimiento entre ellos y el éxito de los proyectos.

La educación mediada por computador (e-learning) permite ofrecer enseñanza de manera distribuida respetando los diferentes ritmos de aprendizaje [4]. Diferentes investigaciones le han integrado técnicas de la inteligencia artificial, con el propósito de construir ambientes de enseñanza-aprendizaje ricos en aspectos pedagógicos y tecnológicos. El principal aporte de la inteligencia artificial queda plasmado en tres variables: adaptación, autonomía y flexibilidad. Uno de estos ambientes son los sistemas tutoriales inteligentes (Intelligent Tutoring Systems, ITS) los cuales permiten ofrecer de manera autónoma enseñanza adaptada y flexible de acuerdo con el perfil cognitivo de cada uno de los estudiantes. Los ITS planifican y replanifican la enseñanza de acuerdo al seguimiento realizado a cada uno de los estudiantes [5]. El Razonamiento basado en casos (Case-Based Reasoning, CBR) es otra de las técnicas y funciona tratando de llegar a la solución de problemas, de manera similar como lo hacemos los seres humanos, es decir, utilizando la experiencia acumulada en situaciones problemáticas similares [6].

El trabajo mostrado en este artículo presenta un método para la formación de stakeholder en proyectos en ingeniería modelado a través de un ITS. El método permite mejorar el éxito en la ejecución de los proyectos. El artículo está distribuido de la siguiente manera: en el capítulo dos se presentan los materiales y métodos haciendo hincapié en los proyectos en ingeniería, stakeholder y las técnicas de la inteligencia artificial (ITS y CBR). El capítulo tres exterioriza los resultados del modelo de ITS para la formación de stakeholder y su respectiva discusión. Finalmente se presentan las conclusiones y la bibliografía.

\section{MATERIALES Y MÉTODOS}

\subsection{Proyectos en ingeniería}

Actualmente existen varias metodologías para la dirección de los proyectos. Las más importantes son Scrum, Prince2 y PMI. El Project Management Institute (PMI) es una de las asociaciones profesionales de miembros más grandes del mundo que cuenta con medio millón de miembros e individuos titulares de sus certificaciones en 180 países. Esta organización americana funciona sin fines de lucro y busca profesionalizar la dirección de proyectos a través de estándares y certificaciones reconocidas mundialmente, por medio de comunidades de colaboración, de un extenso programa de investigación y de oportunidades de desarrollo profesional. Su desarrollo intelectual lo fundamenta el libro PM$\mathrm{BOK}{ }^{\circledR}$ Guide donde expresa toda su metodología 
para el perfecto desarrollo y ejecución de los proyectos. El PMBOK ${ }^{\circledR}$ Guide está compuesto por nueve áreas de conocimiento, cinco grupos de procesos base y 42 procesos. Con esta estructura busca que los proyectos tengan un flujo de trabajo adecuado para ser exitosos [7]. Los cinco grupos de procesos para la dirección de proyectos son [8] (ver figura 1):

\subsubsection{Iniciación: se definen los objetivos del proyecto.}

Se identifican a los principales interesados, se nombra al director y se autoriza formalmente el inicio.

\subsubsection{Planificación:}

Se define el alcance, se refinan los objetivos y se desarrolla el plan para la dirección, que será el curso de acción para que el proyecto sea exitoso.

\subsubsection{Ejecución}

Se integran todos los recursos a los fines de implementar el plan para la dirección.

\subsubsection{Monitoreo y control}

Se supervisa el avance del proyecto y se aplican acciones correctivas.

\subsubsection{Cierre}

Se formaliza con el cliente la aceptación de los entregables del proyecto.
Todos estos procesos están apoyados de nueve áreas de conocimiento, expresados bajo el término de gestión, a saber [8]:

\subsubsection{Integración}

Define los procesos y actividades que integran los diversos elementos de la dirección.

\subsubsection{Alcance}

Muestra los procesos involucrados en garantizar que el proyecto incluya todo (y únicamente) el trabajo requerido para completarlo exitosamente.

\subsubsection{Tiempo}

Se centra en los procesos que se utilizan para garantizar la conclusión a tiempo.

\subsubsection{Costos}

Describe los procesos involucrados en planificar, estimar, presupuestar y controlar los costos de modo que se complete el proyecto dentro del presupuesto aprobado.

\subsubsection{Calidad}

Describe los procesos involucrados en planificar, dar seguimiento, controlar y garantizar que se cumpla con los requisitos de calidad.

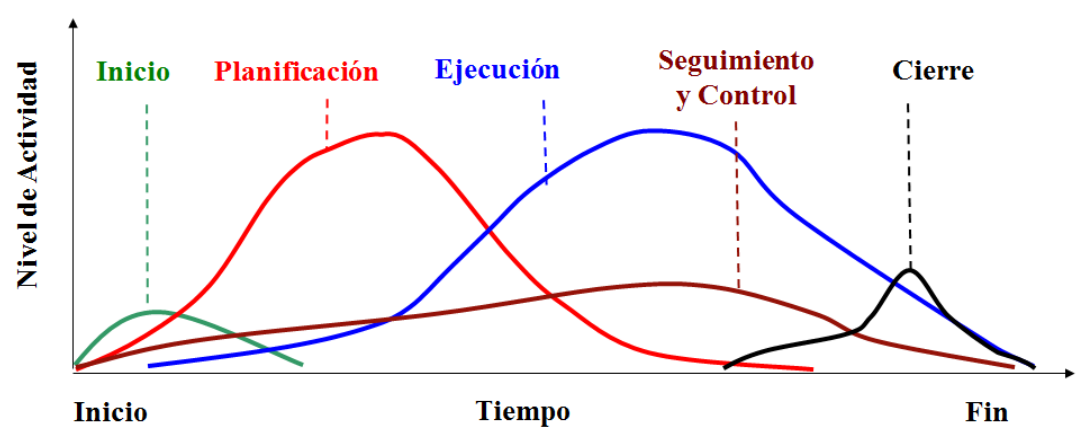

Figura 1. Grupos de procesos [8] 


\subsubsection{Recursos humanos}

Describe los procesos involucrados en la planificación, adquisición, desarrollo y gestión del equipo.

\subsubsection{Comunicaciones}

Identifica los procesos involucrados en garantizar que la generación, recopilación, distribución, almacenamiento y disposición final de la información, sean adecuados y oportunos.

\subsubsection{Riesgos}

Describe los procesos involucrados en la identificación, análisis y control de los riesgos.

\subsubsection{Adquisiciones}

Describe los procesos involucrados en la compra o adquisición de productos, servicios o resultados.

\subsection{Stakeholder}

Stakeholder es definido como el grupo de interés en un proyecto, como por ejemplo las personas, entidades, leyes, medio ambiente, entre otros. Para transmitir conocimiento a múltiples interesados, es importante tener en cuenta los canales de comunicación, la infraestructura social, infraestructura técnica, espacio físico, interacción estilo y contenido. La infraestructura social implica las relaciones sociales entre los ejecutores y los usuarios. La infraestructura técnica comprende la arquitectura de la red, hardware y componentes de software, espacio físico, evaluar el espacio local para la instalación de cámaras, micrófonos, la iluminación el tamaño del salón, tipo de muebles, tamaño de la pantalla, tamaño de la presentación. La interacción estilo y contenido se refiere a los actores que van a participar, cuántos usuarios, contenido tipo de información que se va a trabajar [9], [10].

Todo proyecto en ingeniería es diferente, tiene diferentes circunstancias y requieren de diferentes enfoques. Un proyecto depende de los resulta- dos finales. El principal factor determinante que hace que un proyecto sea diferente a otro son las personas.

Todo proyecto es impulsado por las partes interesadas, por sus deseos, temores, sueños y apuestas que determinan el curso del proyecto. El análisis de los actores es una técnica para identificar y analizar los grupos de interés que rodean a un proyecto; este análisis proporciona información sobre los actores y sus relaciones, intereses y expectativas. Un análisis adecuado de los grupos de interés le ayudará a construir un proyecto y a dar el enfoque adecuado a la situación, y permite además, negociar mejor con el grupo de interés, con los temores hay juego que perder, y con los deseos de que hay algo que ganar [11, 12].

El éxito del proyecto no solo se basa en el nivel de gestión de los administradores del proyecto. Requiere que todos los interesados apoyen y participen del mismo. Los interesados son un factor importante de éxito. La investigación teórica de proyectos indica que los interesados no son capaces de interactuar de una manera ordenada y no tienen una relación armónica; este es un factor importante en la insuficiencia del proyecto. La cooperación de las partes interesadas tiene un impacto importante en el éxito del proyecto. La causa fundamental de fracaso de los proyectos son los intereses de los interesados, y cómo los beneficios del proyecto se distribuyen, es de gran preocupación para todos los socios, ya que la injusta distribución puede dar lugar a malentendidos y conflictos entre partes [12].

\subsection{Inteligencia artificial}

La inteligencia artificial es una disciplina dentro del campo de la informática encargada del estudio del comportamiento del ser humano para trasladarlo a una maquina. Las características humanas estudiadas son: aprendizaje, adaptación, razonamiento, autocorrección, mejoramiento implícito, percepción 
modular del mundo. Algunas de las técnicas son la lógica difusa, el razonamiento basado en casos, el reconocimiento de patrones, las redes neuronales, el sistema de expertos, el aprendizaje de máquina, el tratamiento del lenguaje natural, la robótica, entre otros [13].

La inteligencia artificial en la educación es un campo creciente de interés, donde se trata de aportar en la formulación y aplicación de técnicas al desarrollo de sistemas que soporten los procesos de enseñanza y de aprendizaje, con el propósito de construir sistemas más inteligentes. El término "inteligente" utilizado en estos sistemas queda determinado fundamentalmente por su capacidad de adaptación continua a las características del aprendizaje y del conocimiento de los diferentes usuarios.

Los sistemas tutoriales inteligente (Intelligent Tutoring Systems, ITS) están diseñados para impartir instrucción y apoyar los procesos de enseñanza y de aprendizaje mediante la interacción con el estudiante [14]. Se le da el calificativo particular de "inteligentes" para contrastarlo con los sistemas tradicionales de enseñanaza asistida por computador, siendo la principal diferencia el reconocimiento de la evolución del nivel de aprendizaje de cada uno de los estudiantes.

La arquitectura genérica de los ITS está compuesta por tres módulos, a saber: tutor, estudiante y conocimiento. El primer módulo genera un plan basado en las necesidades específicas del estudiante. Este plan es diseñado para identificar y definir los métodos que ayudan al estudiante a adquirir el conocimiento. Es constantemente replanificado de acuerdo con los hallazgos encontrados. El segundo módulo se encarga de administrar toda la información del estudiante. El tercer módulo almacena los contenidos de la asignatura que se está ofreciendo. Algunos ITS presentan un cuarto módulo llamado Interfaz, el cual se encarga de recibir los datos del estudiante y desplegar la información en la pantalla [15].
El Razonamiento Basado en Casos (Cases-Based Reasoning, CBR) es una técnica que intenta llegar a la solución de nuevos problemas de forma similar a como lo hacen los seres humanos, es decir, utilizando la experiencia acumulada hasta el momento en acontecimientos similares. Un nuevo problema se compara con los casos almacenados previamente en la base de casos y se recuperan uno o varios casos. Posteriormente se utiliza y evalúa una solución sugerida por los casos que han sido seleccionados con anterioridad, para tratar de aplicarla al problema actual. Un caso se compone de tres elementos: la descripción del problema, la solución que se aplicó y el resultado de la solución. Los sistemas CBR son capaces de adquirir nuevo conocimiento a través de la incorporación de casos, y su mantenimiento es más sencillo [16], [17].

\section{RESULTADOSY DISCUSIÓN}

El método propuesto para la formación de interesados (stakeholder) en la ejecución de proyectos de ingeniería se fundamentó en técnicas de la inteligencia artificial. Para lograr lo anterior, se modeló el ambiente de enseñanza y aprendizaje a través de la arquitectura de un ITS. De igual manera, para la selección y visualización de ejemplos presentados a los estudiantes, se tomó como referencia el funcionamiento de un CBR. Se aclara que de ahora en adelante se utilizarán los términos "estudiante" para referirse dentro del stakeholder, solo a las personas interesadas en aprender, y "profesor" a la persona encargada de capacitar. Ambos usuarios pueden ser conocidos también como "actores" y son las entidades externas al sistema [17]. De igual manera, el ambiente de enseñanza y aprendizaje puede ser enfocado como un ambiente instruccional de capacitación o entrenamiento.

Se utilizaron los ITS y CBR en vez de utilizar las plataformas y técnicas comunes del elearning, porque los primeros ofrecen una serie de bondades, como permitir planificar y replanificar los contenidos y 
actividades de acuerdo con el ritmo de aprendizaje de cada uno de los estudiantes, así como también, ofrecer ejemplos similares a la problemática, cuando un estudiante ha encontrado una dificultad. Ambas bondades son regularmente utilizadas por los profesores humanos. A continuación se presentan los casos de uso para cada uno de los actores (estudiante y profesor), la arquitectura modelada a través del ITS y un comparativo de una serie de variables que demuestran las ventajas del método propuesto frente a la enseñanza tradicional.

\subsection{Casos de uso}

Los casos de uso permiten describir las posibles interacciones o usos de un usuario con el sistema $[17,18]$. A continuación se presenta inicialmente, el conjunto de los casos de uso que ocurren para el usuario estudiante el desarrollo de una sesión típica de aprendizaje, luego se exponen los casos de uso para el profesor (ver figura 2).

\subsubsection{Estudiante}

El estudiante puede realizar cuatro casos dentro del ambiente, a saber:

- Iniciar sesión. Este caso de uso le permite al usuario ingresar al ambiente instruccional y realizar todas las operaciones y procesos que se encuentran en su alcance.

- Historia del estudiante. Permite guardar toda la información del estudiante. Dentro de la información se encuentran las veces que ha ingresado al ambiente, el tiempo que se ha demorado en cada una de las sesiones iniciadas, los contenidos que ha visualizado, las actividades que ha realizado, los problemas propuestos que ha concluido de manera individual o colaborativa, las evaluaciones que ha efectuado, el tiempo que se ha demorado en la producción de cada una de las actividades (evaluaciones, contenidos, etc), los resultados de las evaluaciones, las dificultades que ha hallado, preferencias de uso, las páginas que ha visitado, las ayudas que ha necesitado, entre otros. Para algunos autores, esta información es conocida como el perfil cognitivo del estudiante. Esta información permite adaptar los contenidos a las características específicas de cada uno de los estudiantes.

- Mirar los contenidos. Permite que los estudiante visualicen y los contenidos desplegados en la pantalla. Los contenidos también recibe la denominación de objetos de aprendizaje y pueden ser: animaciones, presentaciones, videos, audios, archivos en diferentes formatos, entre otros. Los contenidos se encuentran distribuidos en unidades, de acuerdo al tema tratado.

- Realizar las actividades. Asociados a los contenidos se encuentran las actividades las cuales permiten ejercitar o reforzar los conocimientos adquiridos en el caso anterior. Dentro de las

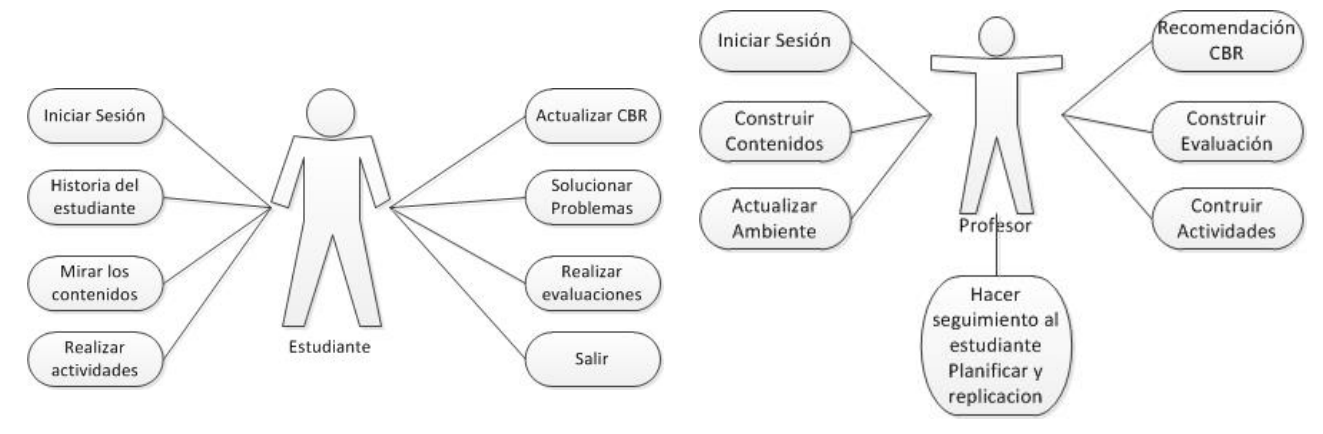

Figura 2. Casos de uso para el estudiante y el profesor.

Fuente: elaboración propia. 
actividades se encuentran, la realización de simulaciones, cuestionarios, ejercicios, participación en foros, redes sociales y construcción de wikis, entre otras.

- Actualizar el CBR. Este caso permite adicionar información nueva a la base de casos del CBR, cuando un estudiante ha encontrado un problema y lo ha solucionado de manera satisfactoria. También permite recuperar un caso, cuando un estudiante encuentra una solución problemática y trata de solucionarla, teniendo en cuenta la experiencia de otros estudiantes en casos similares.

- Solucionar problemas. El ambiente instruccional presenta una serie de problemas propuestos para que el estudiante los solucione de manera individual o colaborativa. El propósito de estos problemas es que el estudiante realice una serie de ejercicios relacionados con los contenidos

- Realizar evaluaciones. En este caso de uso, el estudiante puede realizar cada una de las evaluaciones que el ambiente instruccional le ofrece de acuerdo a los contenidos que se estén abarcando. Cuando los resultados de la evaluación son satisfactorios, la información es registrada en la historia del estudiante. Cuando no logra lo propuesto en la evaluación, la información también es registrada en el historial; sin embargo, se activa el mecanismo de CBR para ofrecerle al estudiante una serie de actividades que otros estudiantes han realizado para tratar de ganar la evaluación.

- Salir. Le permite al estudiante salir del ambiente instruccional.

\subsubsection{Profesor}

El profesor puede realizar siete casos de uso en el ambiente de enseñanza y aprendizaje (ambiente instruccional), a saber:

- Iniciar sesión. Le permite al profesor ingresar al ambiente instruccional.
- Construir contenidos. Le permite al profesor la elaboración de los diferentes objetos de aprendizaje de acuerdo a los temas planteados en cada una de las unidades.

- Actualizar el ambiente. Permite distribuir y renovar los objetos dentro del ambiente. También permite mejorar el concepto visual que utiliza el ambiente.

- Recomendar CBR. Actualiza los casos dentro de la memoria, sacando aquellos que no cumplen las características de la situación problemática del contexto o los perfiles los estudiantes. El profesor también puede sugerir casos para ser utilizados en la memoria del CBR.

- Construir evaluación. Le permite construir los diferentes instrumentos evaluativos de acuerdo con los contenidos de las unidades.

- Construir actividades. Le permite generar diferentes actividades para que sean abordados los contenidos.

- Hacer seguimiento al estudiante (planificar y replanificar). El profesor siempre está atento a cada una de las acciones que realiza el estudiante, con el propósito de sugerirle temas, actividades (ejercicios o colaboración) o guiarlo.

\subsection{Arquitectura}

La arquitectura del ambiente instruccional está conformada por cuatro módulos, cinco bases de datos (incluyendo la base de casos del CBR) y dos tipos de usuarios que pueden realizar las operaciones descritas en los casos de uso. A continuación se presenta la descripción de cada módulo y sus bases de datos (ver figura 3).

\subsubsection{Módulo tutor}

Es el módulo central de la arquitectura. Se encarga de ejercer el control y permitir la planificación y replanificación del aprendizaje del estudiante 


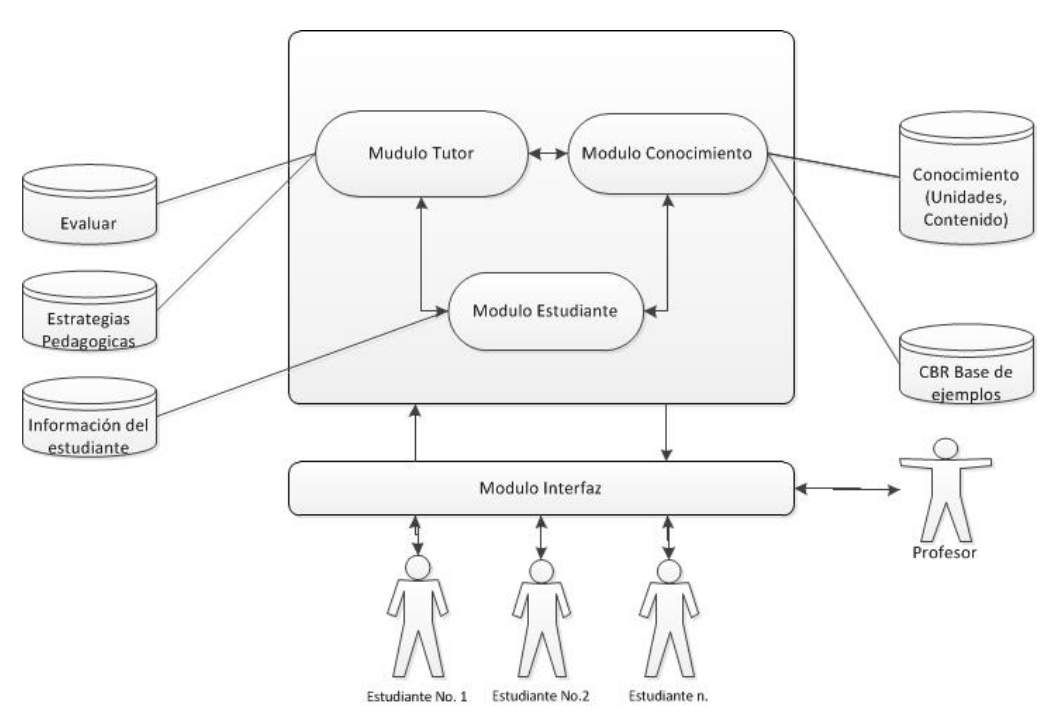

Figura 3. Arquitectura del ambiente de enseñanza y aprendizaje.

Fuente: elaboración propia.

de acuerdo con sus hallazgos. Decide cuándo un estudiante está preparado para presentar una evaluación. Se comunica con los otros módulos para coordinar el correcto funcionamiento del ambiente. Ofrece diferentes estrategias pedagógicas (actividades) para que el estudiante aprehenda los contenidos.

\subsubsection{Módulo conocimientos}

Es el encargado de gestionar los contenidos almacenados en la base de conocimientos a manera de objetos de aprendizaje. También se encarga de la gestión de la base de casos dentro del CBR. Los contenidos y casos son desplegados cuando el módulo tutor los solicita.

\subsubsection{Módulo estudiante}

Es el encargado de gestionar toda la información del estudiante.

\subsubsection{Módulo interfaz}

Su función es la de permitir la comunicación de los usuarios con el ambiente instruccional.

\subsubsection{Base de evaluaciones}

Almacena el conjunto de evaluaciones teniendo en cuenta cada una de las unidades y los diferentes niveles de aprendizaje de los usuarios.

\subsubsection{Base de estrategias pedagógicas}

Contiene el conjunto de actividades propuestas.

\subsubsection{Base de información del estudiante}

Almacena el perfil cognitivo del estudiante.

\subsubsection{Base de conocimientos}

Acopia los objetos de aprendizaje teniendo en cuenta las diferentes unidades.

\subsubsection{Base de casos (CBR)}

Contiene el conjunto de casos que se les presentan a los estudiantes cuando encuentran una situación problémica.

\subsection{Comparativos}

A continuación se presentan una serie características comparativas entre los dos modelos (tradicional versus propuesto). 
Tabla 1. Comparativo del método tradicional de entrenamiento de los stakeholder frente al método propuesto

\begin{tabular}{|c|c|}
\hline Método tradicional & Método propuesto \\
\hline $\begin{array}{l}\text { Materiales: guías impresas, for- } \\
\text { mularios genéricos en internet, } \\
\text { ejemplos, actividades. El origen } \\
\text { de los contenidos es centralizado. } \\
\text { No ofrece ayudas adicionales. }\end{array}$ & $\begin{array}{l}\text { Materiales: ambiente instruccional (con inclusión de técnicas de la inteligencia artificial que } \\
\text { permiten recomendar material y actividades cuando el estudiante ha encontrado dificultades; } \\
\text { además de planificar continuamente el aprendizaje), evaluaciones personalizadas, canales } \\
\text { colaborativos de comunicación, ejemplos y actividades. El estudiante puede acceder al material } \\
\text { en cualquier momento y sin importar el lugar. El origen de los contenidos es distribuido. } \\
\text { Ofrece ayudas adicionales y motivación para buscar otras fuentes de información. Se hace } \\
\text { énfasis en el uso de la tecnología. }\end{array}$ \\
\hline $\begin{array}{l}\text { Profesor: asume el rol de experto } \\
\text { o autoridad formal, transmite la } \\
\text { información a los estudiantes. } \\
\text { Las exposiciones se basan en la } \\
\text { comunicación unidireccional; la } \\
\text { información se transmite a un } \\
\text { grupo de alumnos. La iteración } \\
\text { profesor - estudiante es poca o } \\
\text { nula. }\end{array}$ & $\begin{array}{l}\text { Profesor: es un facilitador-asesor, los estudiantes toman la responsabilidad de aprender. } \\
\text { Incrementan la motivación de los estudiantes presentando problemas reales. Ofrecen retroa- } \\
\text { limentación en las sesiones. La iteración profesor-estudiante es frecuente. }\end{array}$ \\
\hline $\begin{array}{l}\text { Estudiantes: son vistos como } \\
\text { "recipientes vacíos" o receptores } \\
\text { pasivos de información. Ab- } \\
\text { sorben, transcriben, memorizan } \\
\text { y repiten la información para las } \\
\text { evaluaciones. El estudiante puede } \\
\text { asumir responsabilidades. }\end{array}$ & $\begin{array}{l}\text { Estudiantes: son vistos como sujetos que pueden aprender teniendo en cuenta su ritmo de } \\
\text { aprendizaje. Trabajan de manera individual o en equipos para resolver problemas, adqui- } \\
\text { riendo y aplicando el conocimiento en una variedad de contextos. Participan activamente } \\
\text { en la resolución de problema, exploran alternativas de solución, identifican necesidades de } \\
\text { aprendizaje, investigan, aprenden, aplican y resuelven problemas. Se requiere responsabilidad } \\
\text { del estudiante. }\end{array}$ \\
\hline $\begin{array}{l}\text { Evaluación: formularios predefi- } \\
\text { nidos. Los estudiantes buscan la } \\
\text { "respuesta correcta" para tener } \\
\text { éxito en un examen. Se brinda } \\
\text { calificación. Es sumatoria y el pro- } \\
\text { fesor es el único evaluador. No se } \\
\text { pueden repetir las evaluaciones. }\end{array}$ & $\begin{array}{l}\text { Evaluación: tiene en cuenta las características de aprendizaje de cada uno de los estudiantes. } \\
\text { Se brinda calificación y retroalimentación personalizada. Se ofrecen alternativas de remedia- } \\
\text { ción cuando el estudiante ha perdido una evaluación (CBR). Permite repetir las evaluaciones. } \\
\text { Existen simulacros preparatorios para las evaluaciones. Además, el profesor implementa una } \\
\text { evaluación integral, en la que es importante tanto el proceso como el resultado y está com- } \\
\text { puesta por: una evaluación individual, una colaborativa (efectuada por los demás miembros } \\
\text { del grupo de estudiantes), una autoevaluación (el estudiante evalúa su propio proceso) y una } \\
\text { coevaluación (realizada entre el estudiante y profesor), todo en un mismo escenario. }\end{array}$ \\
\hline $\begin{array}{l}\text { Aprendizaje: es individual y de } \\
\text { competencia. }\end{array}$ & $\begin{array}{l}\text { Aprendizaje: utilizan el ambiente instruccional rico en recursos, donde además pueden } \\
\text { experimentar con diversos recursos con retroalimentación inmediata. }\end{array}$ \\
\hline $\begin{array}{l}\text { Paradigma instruccional: } \\
\text { clases magistrales basadas en el } \\
\text { constructivismo o conductismo. }\end{array}$ & Paradigma instruccional: integración de conductismo, constructivismo e histórico-social. \\
\hline Centro del proceso: profesor. & Centro del proceso: estudiante. \\
\hline $\begin{array}{l}\text { Retroalimentación: el estudiante } \\
\text { puede preguntar en las sesiones } \\
\text { presenciales. }\end{array}$ & $\begin{array}{l}\text { Retroalimentación: en cualquier momento cuando el estudiante lo requiere. Al finalizar } \\
\text { una evaluación le ofrece información de retorno y una serie de contenidos y actividades si el } \\
\text { estudiante no alcanzó lo propuesto. }\end{array}$ \\
\hline
\end{tabular}




\section{CONCLUSIONES}

Cuando existen contratiempos dentro del tiempo de la ejecución de los proyectos en ingeniería, regularmente los stakeholder argumentan que la culpa la tiene el aspecto financiero del mismo. Sin embargo, la dificultad puede que no tenga sus raíces en el aspecto financiero sino en la formación misma de los stakeholder, sin dejar a un lado diversas variables como la programación de la ejecución del proyecto o la concienciación de las diversas variables del problema a atacar, entre otras.

Dentro del entrenamiento o formación de los stakeholder se encuentran diversas ambigüedades. Uno de ellas es la falta de aprehensión, profundización y concientización en los contenidos educativos y los grupos de procesos después de realizado un proceso de entrenamiento. Los stakeholder en ocasiones no tienen una idea global de la transformación que se está adelantando; de igual manera desconocen las fases iniciales como la identificación de la problemática a enfrentar y elicitación de sus requisitos.

En los procesos educativos, la continua innovación, la inclusión de las didácticas y una excelente actitud de los actores son factores obligatoriamente necesarios para lograr mejores resultados en la transmisión y apropiación del conocimiento.

En este artículo se presentó un método para la formación de stakeholder en la ejecución de proyectos de ingeniería. El método fue modelado a través de un ITS y se utilizó además la técnica del CBR, ofreciendo ventajas significativas frente a métodos tradicionales de instrucción en empresas.

Dentro de los resultados, se puede apreciar que el reforzamiento continuo propio del CBR cuando un estudiante no alcanza lo propuesto en un tema, permite una mayor profundización de los contenidos. De igual manera, el ambiente instruccional no es un ambiente plano, sino que siempre tiene en cuenta las características propias de los estudiantes para planificar y replanificar continuamente las actividades que se están enseñando. La utilidad en la formación de proyectos de ingeniería es evidente debido a que se logra una mayor aprehensión y conscienciación de los contenidos y grupos de procesos por parte de los stakeholder.

Es imperiosamente necesario cambiar los métodos tradicionales de enseñanza, pero primero debe de hacerse una fuerte labor de concientización a los actores del proceso educativo referente al cambio de paradigma.

\section{REFERENCIAS}

[1] Ciocoiu, L., Henshaw, M. J. D. C., \& Hubbard, E. M. (2012). A systems - of-systems approach to the development of flexible, cost-effective training environments. In System of Systems Engineering (SoSE), 2012 7th International Conference on (pp. 531-536). IEEE.

[2] Li, X., \& Mingsheng, S. (2010). The Research on Benefit Coordination Mechanism of Project Stakeholder. In Computational Intelligence and Design (ISCID), 2010 International Symposium on (Vol. 2, pp. 248-251). IEEE.

[3] Huang, J., \& Sun, J. (2009). The Provision of Incentives in the Construction Projects. In Management and Service Science, 2009. MASS'09. International Conference on (pp. 1-4). IEEE.

[4] Saowapakpongchai, K. (2010). The development of elearning model for higher education in Thailand. Educational and Network Technology (ICENT), 2010 International Conference on pp.16,19, 25-27.

[5] Wang, L.; Lu, W.; Wang, C. (2012). Research on Key Technology of Cheerleading Intelligent Tutoring System Design. In Computer Science \& Education (ICCSE), 2012 7th International Conference on (pp. 1498-1501). IEEE.

[6] Zhou, P.; Yin, W.; Zhao, J. (2012). Research on case retention strategy for industrial case-based reasoning (CBR) system: A practical case study. International Conference on Computer Science and Information Processing (CSIP), 2012. Page(s): 376 - 380. IEEE Conference Publications.

[7] PMI (2013) Proyect Manager Institute. [En línea http:// www.pmi.org/], fecha de Acceso Mayo de 2013.

[8] PMBOK (2008). Guía de los Fundamentos para la Dirección de Proyectos. Atlanta: Project Management Institute. 
[9] Barfurth, M. A., Singer, J., Emond, B., Vinson, N., Brooks, M., \& Spence, J. (2002). Evaluation factors for multi-stakeholder broadband visual communication projects. In Enabling Technologies: Infrastructure for Collaborative Enterprises, 2002. WET ICE 2002. Proceedings. Eleventh IEEE International Workshops on (pp. 18-22). IEEE.

[10] Sundstrom, P. (2004). Opportunities for creativity in project work-importance of goals and problem solving. In Engineering Management Conference, 2004. Proceedings. 2004 IEEE International (Vol. 3, pp. 978-983). IEEE.

[11] De Baar, B. (2006). Using Stakeholder Analysis in Software Project Management.

[12] Lu, X., Jia, Z., Liu, W., \& Wang, F. (2008, October). Simulation study of multi-agent-based network manufacturing ASP system. In Service Operations and Logistics, and Informatics, 2008. IEEE/SOLI 2008. IEEE International Conference on (Vol. 2, pp. 1849-1853). IEEE.

[13] Russell, S.; Norving, P. (2004) Intelligent Agents. En: Artificial Intelligence: A Modern Approach. Second Edition. Prentice Hall Series in Artificial Intelligence.
[14] Caro, M.; Jiménez, J.; Paternina, A. (2012) Architectonical Modeling of Metamemory Judgment in Case Based Reasoning Systems. En: XXXVIII Conferencia Latinoamericana en Informática CLEI 2012 / Simposio en Investigación de Operaciones e Inteligencia Artificial.

[15] Ramesh, V. M., \& Rao, N. J. (2012). Tutoring and Expert Modules of Intelligent Tutoring Systems. In Technology for Education (T4E), 2012 IEEE Fourth International Conference on (pp. 251-252). IEEE.

[16] Cheng, P., Zhao, K., Li, Y., \& Xu, W. (2011). Application of case based reasoning in plane geometry intelligent tutoring system. In Electrical and Control Engineering (ICECE), 2011 International Conference on (pp. 4369-4373). IEEE.

[17] Chunyan, Q. (2010). UML-based software process modeling. In Computer, Mechatronics, Control and Electronic Engineering (CMCE), 2010 International Conference on (Vol. 1, pp. 247 -250). IEEE.

[18] Jiménez, J.; Gaviria, J.; Arevalo, W. (2011) Building virtual learning objects that accomplish today CBR system requirements. Software Engineering: Methods, Modeling, and Teaching. En: Colombia. Ed: Sello Editorial Universidad de Medellín, v. 1, pp. 103-112. 